\title{
TERAPIA COGNITIVO-COMPORTAMENTAL DE GRUPO NO TRATAMENTO DA FOBIA SOCIAL GENERALIZADA
}

\author{
Cognitive-Behavioral Group Therapy in the Treatment \\ of Generalized Social Phobia
}

GustavoJ.FonsecaD'ElRey ${ }^{l}$

Paloma N. Greenberg ${ }^{2}$

Mohamed Abdallah Husni ${ }^{3}$

Adriana Cejkinski ${ }^{4}$

\section{Resumo}

Este estudo teve como objetivo verificar a efetividade da terapia cognitivo-comportamental de grupo em pacientes com fobia social generalizada. Vinte e nove pacientes com diagnóstico de fobia social generalizada foram randomicamente distribuídos em dois grupos de tratamento. Um grupo de terapia cognitivo-comportamental de grupo (TCCG) e outro grupo de controle placebo com credibilidade (CPC). Os pacientes preencheram 3 escalas de avaliação (Inventário de Fobia Social, Inventário de Ansiedade Beck e Escala de Impressão Clínica Global) na semana 1 de tratamento e na semana 12. Em todas as medidas de avaliações, ao final de 12 semanas, os pacientes que receberam TCCG apresentaram melhoras superioras ao grupo de CPC. Neste ensaio clínico randomizado, simples-cego, a TCCG foi superior ao CPC no tratamento da fobia social generalizada.

Palavras-chave: Fobia social; Psicoterapia de grupo; Terapia cognitivo-comportamental de grupo; Tratamento.

\footnotetext{
Psicólogo. Especialista em Psicologia Hospitalar pela Faculdade de Medicina da USP. Formação em Terapia Cognitivo-Comportamental pelo CEPESP. Aprimoramento em Psicopatologia pela Faculdade de Psicologia da USJT. Coordenador do Programa de Fobia Social do Centro de Pesquisas e Tratamento de Transtornos de Ansiedade - São Paulo, SP.

2 Psicóloga. Formação em Terapia Cognitivo-Comportamental pelo CEPESP. Pesquisadora do Programa de Fobia Social do Centro de Pesquisas e Tratamento de Transtornos de Ansiedade - São Paulo, SP.

3 Psiquiatra. Ph.D. em Psicofarmacologia Clínica pela Universidade de Londres. Formação em Terapia Cognitivo-Comportamental pelo CEPESP. Vice-Coordenador do Programa de Fobia Social do Centro de Pesquisas e Tratamento de Transtornos de Ansiedade São Paulo, SP.

4 Psicóloga. Formação em Terapia Cognitivo-Comportamental pelo CEPESP. Pesquisadora do Programa de Fobia Social do Centro de Pesquisas e Tratamento de Transtornos de Ansiedade - São Paulo, SP. e-mail: g.delrey@bol.com.br
} 


\section{Abstract}

The aim of this study is to assess the effectiveness of cognitive-behavioral group therapy in patients with generalized social phobia. Twenty nine patients with diagnosis of generalized social phobia were randomly allocated in two groups of treatments. One group of cognitive-behavioral group therapy (CBGT) and another group of credible placebo control (CPC). The patients completed 3 evaluation scales (Social Phobia Inventory, Beck Anxiety Inventory and Clinical Global Impression Scale) in the weeks 1 and 12. In all evaluation scales in the week 12 of treatment, the CBGT patients were rated as more improved than CPC patients. In this randomized single-blind clinical trial, the CBGT was superior to a CPC in the treatment of generalized social phobia.

Keywords: Social phobia; Group psychotherapy; Cognitive-behavioral group therapy; Treatment.

\section{Introdução}

Confundida com a timidez, a fobia social é muito mais do que isso. É um transtorno mental severo que traz sofrimento e perdas de oportunidades para seu portador (D'El Rey \& Freedner, 2006; Stein \& Kean, 2000).

Diversos tratamentos cognitivocomportamentais e farmacológicos demonstraram sua eficácia e estão atualmente disponíveis para os pacientes com fobia social (D'El Rey \& Pacini, 2006; Gould, Buckminster, Pollack \& Yap, 1997).

Embora diversos estudos tenham demonstrado a eficácia dos tratamentos psicológicos na fobia social, cada vez menos pacientes vêm recebendo esta modalidade de tratamento (D'El Rey \& Pacini, 2005; Fava, Grand, Rafanelli, Conti \& Belluard, 2001; Goisman, Warshaw \& Keller, 1999; Taylor, 1996).

Existem na literatura internacional, diversos estudos controlados que demonstram a eficácia da terapia cognitivo-comportamental em grupo (TCCG). A TCCG produz melhoras superiores a controles na lista de espera e a tratamentos com placebos psicológicos (Hope, Heimberg \& Brush, 1995; Heimberg et al, 1990). A TCCG mantém seus ganhos em follow-ups de 5 anos após o tratamento ter sido descontinuado (Heimberg, Salzman \& Holt, 1993).

Até onde se sabe, aqui no Brasil existem apenas dois estudos relacionados à terapia de grupo no tratamento da fobia social generalizada. Um estudo descreve a terapia psicodinâmica de grupo no tratamento da fobia social generalizada e o outro estudo descreve a comparação entre técnicas (treinamento de habilidades sociais versus treinamento de habilidades sociais acrescido da reestruturação cognitiva) no tratamento deste transtorno (Knijnik, Kapczinski, Chachamovich, Margis \& Eizirik, 2004; D’El Rey, Beidel \& Pacini, 2006).

\section{Objetivo}

Este estudo teve como objetivo avaliar a efetividade da terapia cognitivo-comportamental em grupo (TCCG), em pacientes com fobia social generalizada.

\section{Metodologia}

- Participantes: A amostra compreendeu 29 sujeitos, sendo 18 homens e 11 mulheres, com a média de idade de 36,5 anos (ver Tabela 1). Os pacientes foram recrutados por meio de anúncios em jornais do bairro. Trinta e sete pessoas responderam aos anúncios nos jornais. Oito pessoas não puderam ser incluídas no presente estudo, devido aos critérios estabelecidos para a inclusão, que foram: ter entre 18 e 60 anos; diagnóstico primário de fobia social segundo o DSM-IV; não ter nenhum problema com álcool e drogas; não apresentar escore acima de 16 pontos (sintomas depressivos leves) no Inventário de Depressão Beck; não preencher critérios para outro transtorno mental; não estar recebendo nenhuma forma de tratamento psicoterápico ou psicofarmacológico durante o estudo. Todas as pessoas incluídas neste estudo foram avaliadas para o diagnóstico de fobia social mediante Entrevista Clínica Estruturada para o DSM-IV-SCID-I/P 2.0, reportando altos níveis de ansiedade social e evitação de diversas situações sociais e de desempenho, com interferência nas rotinas diárias e sofrimento (Tavares, 1996; A.P.A., 1995). Cabe ressaltar, que toda a amostra $(n=29)$ deste estudo recebeu o diagnóstico de fobia social generalizada. 
- Procedimentos: As pessoas foram distribuídas randomicamente em dois grupos de tratamento, ou seja, grupo de Terapia CognitivoComportamental em Grupo (TCCG) e Grupo de Controle Placebo com Credibilidade (CPC). Existiram dois grupos por condição. Os grupos foram tratados em 12 sessões semanais de 90 minutos. Após as pessoas terem sido avaliadas para a inclusão no estudo e distribuídas nos grupos de tratamento, foram avaliadas em uma segunda entrevista para o pré-tratamento por meio de 3 escalas de avaliação (descritas a seguir no texto). Ao final do tratamento de 12 semanas, as pessoas foram novamente avaliadas pelas mesmas escalas. Dois psicoterapeutas com experiência (na época do estudo, 7 anos) em tratamento da fobia social conduziram as sessões de tratamento. O primeiro autor acompanhou o grupo de TCCG e o segundo autor acompanhou o grupo de CPC. Durante o período do presente estudo, os dois terapeutas não discutiram o andamento dos tratamentos de ambos os grupos, para que não ocorresse uma contaminação de informações.

a) Terapia Cognitivo-Comportamental de Grupo (TCCG): Nesse estudo, utilizou-se o formato de TCCG descrita pelo Dr. Richard G. Heimberg do Departamento de Psicologia da Temple University e seu grupo (Heimberg, Juster, Hope \& Mattia, 1995). Integram-se técnicas de exposição e reestruturação cognitiva neste modelo de tratamento. A TCCG foi administrada em grupos de aproximadamente 7 pacientes em 12 sessões (uma por semana) com duração de 90 minutos. Nas sessões 1 e 2, os pacientes receberam informações sobre a fobia social e instruções para a exposição, reestruturação cognitiva e tarefas para casa, bem como praticaram as habilidades de reestruturação cognitiva recém-aprendidas. Nas sessões seguintes, o terapeuta trabalhou com os pacientes individualmente na exposição às situações sociais temidas por cada um. Durante toda a exposição, o terapeuta orientou a reestruturação cognitiva. Os pacientes foram orientados a corrigirem as distorções cognitivas que aparecessem durante a exposição. Ao final de cada sessão, os pacientes comentaram sobre as tarefas de casa realizadas durante a semana. As tarefas de casa consistiam tipicamente na exposição na vida real das situações que foram dissensibilizadas nas sessões de terapia. b) Grupo de Controle Placebo com Credibilidade (CPC): O CPC é um conjunto de procedimentos padronizados desenvolvidos pelo Dr. Heimberg e seu grupo de pesquisa (Heimberg et al., 1990). Esses procedimentos foram criados como uma forma de comparação com tratamentos psicológicos ativos, semelhante aos ensaios clínicos placebocontrolados randomizados realizados utilizando fármacos. O CPC combina psicoterapia de apoio de grupo e apresentações educativas. Durante a primeira sessão de tratamento, foram focados assuntos relativos às bases do tratamento da fobia social. Nos primeiros 45 minutos das sessões 2 a 12, foram apresentadas uma série de aulas (discussões) sobre aspectos relevantes às pessoas portadoras de fobia social. Nos 45 minutos finais das sessões 2 a 12, as pessoas do grupo compartilhavam suas atividades semanais e as preocupações em relação a eventos futuros provocadores de ansiedade social. Eles também sugeriram métodos por eles utilizados para lidar com situações que são comuns a outras pessoas do grupo. Diferentemente da TCCG, o terapeuta não forneceu instruções específicas para os pacientes se exporem a situações geradoras de ansiedade social (técnica de exposição). Também não foi corrigida nenhuma percepção distorcida dos pacientes em relação a eventos geradores de ansiedade (reestruturação cognitiva).

- Medidas de avaliação: No Prétratamento - Semana 1 e no Pós-tratamento - Semana 12, foram utilizadas as seguintes medidas de avaliações:

a) Inventário de Fobia Social (SPIN): O SPIN é um instrumento que consiste de 17 itens. Para cada item do questionário, solicita-se ao indivíduo que indique o quanto as situações o incomodaram, devendo marcar uma entre as 05 opções, que variam de "de forma alguma à extremamente". A pontuação para cada uma das opções varia de 0 a 4 , e a pontuação total do inventário varia de 0 a 68. Escores de 19 pontos ou mais indicam a presença de sintomas compatíveis com o diagnóstico de fobia social (Vilete, Coutinho \& Figueira, 2004).

b) Inventário de Ansiedade Beck (BAI): O BAI é composto por 21 itens, com alternativas de respostas variando entre "nada à gravemente". A pontuação total do inventário varia de 0 a 63 . A classificação recomendada para a ansiedade é 0-7 = ansiedade mínima; 8-15 = ansiedade leve; $16-25=$ ansiedade moderada; e 26-63 = ansiedade grave (Cunha, 2001). 
c) Escala de Impressão Clínica Global (CGIS): A CGIS avalia o grau de interferência de sintomas psicopatológicos no funcionamento do paciente. Este instrumento é baseado em uma escala de 5 pontos, ou seja, $1=$ sem sintomas; $2=$ alguns sintomas estão presentes, mas eles não interferem no trabalho ou atividades sociais; $3=$ sintomas interferem um pouco no trabalho ou em atividades sociais; $4=$ sintomas interferem muito no trabalho e atividades sociais; e $5=$ as atividades de trabalho e sociais estão seriamente comprometidas pelos sintomas. O escore total varia de 1 a 5 (Cooper, 1963).

- Análise estatística: Os escores dos instrumentos de avaliação foram expressos em média e desvio-padrão. Análises de covariância (ANCOVA) e multivariada da variância (MANOVA) foram utilizadas ao longo dos resultados deste estudo. O nível de significância estatística foi de $5 \%$. Para desenvolver as análises dos dados, foi utilizado o programa Statistica edição 99.
- Questões éticas: Este estudo esteve de acordo com a Resolução do Conselho Nacional de Saúde (196/96) para pesquisa envolvendo seres humanos. A presente pesquisa foi aprovada pelo Comitê de Ética em Pesquisa do Centro de Pesquisas e Tratamento de Transtornos de Ansiedade - São Paulo-SP.

\section{Resultados}

\section{a) Características da amostra}

As características dos pacientes no Prétratamento - Semana 1 estão na Tabela 1. Conforme a análise multivariada da variância (MANOVA), não existiram diferenças significativas entre os dois grupos no início do tratamento em relação aos dados sociodemográficos (lambda de Wilks = 0,95; $\left.F_{1,27}=3,76 ; p=0,021\right)$ e em relação aos escores dos instrumentos de avaliação (lambda de Wilks = 0,$\left.78 ; F_{1,27}=2,34 ; p=0,036\right)$. Todos os 29 pacientes terminaram o estudo.

\section{Tabela 1 - Características da amostra $(n=29)$}

\begin{tabular}{|c|c|c|}
\hline Características & $\begin{array}{l}\text { TCCG } \\
(\mathrm{n}=15)\end{array}$ & $\begin{array}{l}\text { CPC } \\
(n=14)\end{array}$ \\
\hline \multicolumn{3}{|l|}{ Sexo } \\
\hline Masculino & 10 & 8 \\
\hline Feminino & 5 & 6 \\
\hline \multicolumn{3}{|l|}{ Idade } \\
\hline Média em anos & 36,9 & 36,1 \\
\hline Desvio padrão & 4,9 & 5,3 \\
\hline \multicolumn{3}{|l|}{ Estado civil } \\
\hline Solteiro & 11 & 11 \\
\hline Casado & 4 & 3 \\
\hline \multicolumn{3}{|l|}{ Ocupação atual } \\
\hline Empregado & 12 & 11 \\
\hline Desempregado & 2 & 3 \\
\hline Estudante & 1 & 0 \\
\hline
\end{tabular}

Notas: TCCG = Terapia cognitivo-comportamental; $\mathrm{CPC}=$ Controle placebo com credibilidade. 


\section{b) Comparação entre os tratamentos (TCCG X CPC)}

Segundo a análise de covariância (ANCOVA) realizada para cada uma das três medidas de avaliação (SPIN: $F_{1,26}=80,55 ; p=$ 0,0001 / BAI: $F_{1,26}=113,12 ; p=0,00001 /$ CGIS: $\left.F_{1,26}=15,23 ; p=0,001\right)$, o grupo de TCCG apresentou uma melhora significativamente superior ao grupo de CPC, ou seja, ocorreu uma redução significativa nos escores das escalas de avaliação, como pode ser observado na Tabela 2.

Conforme o Inventário de Fobia Social, 5 participantes do grupo de TCCG $(33,3 \%$ da amostra de 15 pacientes e $17,2 \%$ da amostra total de 29 pacientes) ao final das 12 semanas apresentavam escores inferiores a 19 pontos, ou seja, não apresentavam mais sintomas fóbicos sociais.

Em relação à Escala de Impressão Clínica Global, o grupo que recebeu a TCCG ao final das 12 semanas de tratamento teve como média o escore 2 (alguns sintomas estão presentes, mas eles não interferem no trabalho ou atividades sociais), enquanto que a média ao final do estudo no grupo CPC foi 3 (sintomas interferem um pouco no trabalho ou em atividades sociais).

\section{Tabela 2 - Escores de ambos os grupos nas medidas de avaliação (média \pm DP)}

\begin{tabular}{lcccc}
\hline \multicolumn{2}{c}{ Semana 1 } & & Semana 12 & \\
Escalas & TCCG & CPC & TCCG & CPC \\
& & & & \\
\hline SPIN & $41 \pm 4,3$ & $38 \pm 4,0$ & $23 \pm 5,8$ & $31 \pm 3,8$ \\
BAI & $25 \pm 8,5$ & $24 \pm 8,3$ & $12 \pm 4,2$ & $19 \pm 4,7$ \\
CGIS & $\mathbf{4} \pm \mathbf{1 , 2}$ & $\mathbf{4} \pm \mathbf{0 , 9}$ & $\mathbf{2} \pm \mathbf{1 , 0}$ & $3 \pm \mathbf{0 , 7}$ \\
\hline
\end{tabular}

Notas: TCCG $=$ Terapia cognitivo-comportamental; $\mathrm{CPC}=$ Controle placebo com credibilidade DP $=$ Desviopadrão; SPIN = Inventário de fobia social; BAI = Inventário de ansiedade Beck; CGIS = Escala de impressão clínica global.

\section{Discussão}

Até onde se sabe, não existem aqui no Brasil estudos comparando a terapia cognitivocomportamental de grupo (TCCG) com um grupo de controle placebo com credibilidade (CPC) no tratamento da fobia social generalizada.

O grupo de TCCG apresentou melhoras significativamente superiores ao grupo CPC, como pode ser visto pelas medidas de avaliações.

Observando-se o Inventário de Fobia Social (SPIN), que avalia 3 critérios importantes que definem o quadro clínico da fobia social, ou seja, o medo, a evitação das situações e os sintomas somáticos; a diminuição nos escores no grupo de TCCG foram significativamente superiores ao grupo controle, indicando uma diminuição nos sintomas fóbicos sociais destes pacientes (Vilete et al., 2004). Cinco pacientes do grupo de TCCG, ao final das 12 semanas, apresentavam escores inferiores a 19 pontos, ou seja, segundo esta medida de avaliação, não apresentavam mais sintomas compatíveis com o quadro clínico de fobia social. Acredita-se que se este estudo fosse estendido por mais semanas, outros pacientes deste grupo poderiam apresentar escores inferiores a 19 pontos.

Conforme a Escala de Impressão Clínica Global (CGIS), a TCCG foi um tratamento que trouxe melhoras ao funcionamento dos pacientes a ela submetida, ou seja, os sintomas fóbicos sociais passaram a influenciar menos suas atividades diárias. Isso pode ser corroborado pelo Inventário de 
Ansiedade Beck (BAI), pois com a diminuição dos sintomas de ansiedade, a funcionalidade dos pacientes melhorou. Conforme Clark et al (2003), a melhora nos sintomas ansiosos leva geralmente a uma melhora nas rotinas diárias dos pacientes acometidos pela fobia social.

Outro fator que deve ser levado em conta é o próprio fator grupo, pois este favorece uma maior exposição aos pacientes fóbicos sociais, uma vez que eles evitam atividades que são realizadas na presença de outras pessoas (D'El Rey \& Pacini, 2006; Heimberg et al., 1995).

Este estudo encontrou resultados semelhantes ao estudo de Heimberg et al. (1990), ou seja, de que a TCCG é superior ao tratamento com CPC na fobia social generalizada.

Embora os resultados da literatura sugiram que a TCCG e a farmacoterapia sejam tratamentos bem estabelecidos para a fobia social, observa-se na prática clínica que nem todos os pacientes respondem favoravelmente a estas modalidades de tratamento, principalmente os fóbicos sociais que apresentam comorbidades com outros transtornos mentais (Gould et al., 1997; Chambless, Tran \& Glass, 1997). Outras modalidades de tratamento são necessárias para a fobia social. Um estudo que comparou a terapia psicodinâmica de grupo com um grupo de controle placebo com credibilidade (semelhante a este estudo), mostrou que a primeira foi um tratamento também superior ao grupo de CPC na fobia social generalizada (Knijnik et al., 2004).

Infelizmente não se pode fazer uma comparação entre as terapias cognitivocomportamental e psicodinâmica de grupo no tratamento da fobia social generalizada, pois não existe este tipo de estudo. Este é um campo promissor para futuras pesquisas.

As evidências encontradas neste estudo sugerem que a TCCG é um tratamento que deve ser empregado na assistência a pacientes com fobia social, para que os efeitos negativos que este grave transtorno de ansiedade impõem a seu portador sejam minimizados (D'El Rey \& Pacini, 2006; Heimberg, 2001; Stein \& Kean, 2000).

\section{Considerações finais}

Neste estudo de 12 semanas, a terapia cognitivo-comportamental de grupo (TCCG) mostrou-se como um tratamento superior ao realizado com um grupo controle placebo de credibilidade (CPC) na fobia social generalizada.

Sugere-se que outros estudos com desenhos diferentes e com um maior número de pacientes sejam realizados, para a confirmação dos dados aqui encontrados.

\section{Referências}

Associação Psiquiátrica Americana. (1995). DSMIV: Manual diagnóstico e estatístico de transtornos mentais (4a ed.). Porto Alegre: Artes Médicas.

Chambless, D. L., Tran, G. Q., \& Glass, C. R. (1997). Predictors of responde to cognitivebehavioral group therapy for social phobia. Journal of Anxiety Disorders, 11(3), 221-240.

Clark, D. M., Ehlers, A., McManus, F., Hackmann, A., Campbell, H., Flower, T. et al. (2003). Cognitive-behavioral therapy versus fluoxetine in generalized social phobia: a randomized placebo-controlled trial. Journal of Consulting and Clinical Psychology, 71(6), 1058-1067.

Cooper, J. E. (1963). A study of behaviour therapy in 30 psychiatric patients. Lancet, 1(5), 411-415.

Cunha, J. A. (2001). Manual em português das escalas Beck. São Paulo: Casa do Psicólogo.

D’El Rey, G. J. F., Beidel, D. C., \& Pacini, C. A. (2006). Tratamento da fobia social generalizada: comparação entre técnicas. Revista Brasileira de Terapia Comportamental e Cognitiva, 8(1), 1-12.

D’El Rey, G. J. F., \& Freedner, J. J. (2006). Depressão em pacientes com fobia social. Psicologia Argumento, 24(46), 71-76.

D’El Rey, G. J. F., \& Pacini, C. A. (2006). Terapia cognitivo-comportamental da fobia social: modelos e técnicas. Psicologia em Estudo, 11(2), 269-275.

D’El Rey, G. J. F., \& Pacini, C. A. (2005). Tratamento da fobia social por exposição ao vivo e reestruturação cognitiva. Revista de Psiquiatria Clínica, 32(4), 231-235.

Fava, G. A., Grand, S., Rafanelli, C., Conti, S., \& Belluard, P. (2001). Long-term outcome of social phobia treated by exposure. Psychological Medicine, 31(5), 899-905. 
Goisman, R. M., Warshaw, M. G., \& Keller, M. B. (1999). Psychosocial treatment prescriptions for generalized anxiety disorder, panic disorder and social phobia, 1991-1996. American Journal of Psychiatry, 156(11), 1819-1821.

Gould, R. A., Buckminster, A., Pollack, P. M., \& Yap, R. T. (1997). Cognitive-behavioral and pharmacological treatment for social phobia: a meta-analysis. Clinical Psychology: Science and Practice, 4(3), 296-306.

Heimberg, R. G. (2001). Current status of psychotherapeutic interventions for social phobia. Journal of Clinical Psychiatry, 62(Suppl 2), 36-42.

Heimberg, R. G., Dodge, C. S., Hope, D. A., Kennedy, C. R., Zollo, L. J., \& Becker, R. E. (1990). Cognitive-behavioral group treatment for social phobia: comparison with a credible placebo control. Cognitive Therapy and Research, 14(1), 1-23.

Heimberg, R .G., Juster, H. R., Hope, D. A., \& Mattia, J. I. (1995). Cognitive-behavioral group treatment: description, case presentation, and empirical support. In M. B. Stein (Org.), Social phobia: Clinical and research perspectives (pp. 293-321). Whashington, DC: American Psychiatric Press.

Heimberg, R. G., Salzman, D. G., \& Holt, C. S. (1993). Cognitive-behavioral group treatment for social phobia: effectiviness of five-years follow-up. Cognitive Therapy and Research, 17(3), 325-339.

Hope, D. A., Heimberg, R. G., \& Brush, M. A. (1995). Dismantling cognitive-behvioral group therapy for social phobia. Behaviour Research and Therapy, 33(6), 637-650.

Knijnik, D. Z., Kapczinski, F., Chachamovich, E., Margis, R., \& Eizirik, C. L. (2004). Psicoterapia psicodinâmica em grupo para fobia social generalizada. Revista Brasileira de Psiquiatria, 26(2), 77-81.

Stein, M. B., \& Kean, Y. M. (2000). Disability and quality of life in social phobia: epidemiologic findings. American Journal of Psychiatry, 157(10), 1606-1613.

Tavares, M. (1996). Entrevista clínica estruturada para o DSM-IV: Transtornos do eixo I - edição para pacientes (SCID-I/P 2.0). Brasília: Instituto de Psicologia, Universidade de Brasília.
Taylor, S. (1996). Meta-analysis of cognitivebehavioral treatments for social phobia. Journal of Behaviour Therapy and Experimental Psychiatry, 27(1), 1-9.

Vilete, L. M. P., Coutinho, E. S. F., \& Figueira, I. L. V. (2004). Confiabilidade da versão em português do inventário de fobia social (SPIN) entre adolescentes estudantes do município do Rio de Janeiro. Cadernos de Saúde Pública, 20(1), 89-99.

Recebido em: 14/03/2007

Received in: 03/14/2007

Aprovado em: 28/03/2007 Approved in: 03/28/2007 